in: Rauterberg, M. (ed.): Proceedings of the Third International Conference on Educational Computing (ICEC 2004), September 1-3, 2004, Eindhoven, NL, Springer, LNCS, pp. 80-89

\title{
Computer Supported Collaborative Sports: Creating Social Spaces Filled with Sports Activities
}

\author{
Volker Wulf ${ }^{1,3}$, Eckehard F. Moritz ${ }^{2}$, Christian Henneke ${ }^{2}$, Kanan Al-Zubaidi ${ }^{1}$, \\ Gunnar Stevens ${ }^{1}$ \\ ${ }^{1}$ Institute for Information Systems, University of Siegen, Germany \\ \{wulf, kanan, stevens\}@fb5.uni-siegen.de \\ ${ }^{2}$ SportKreativWerkstatt, Technical University of Munich, Germany \\ efmesportkreativwerkstatt.de, christian.henneke@web.de \\ ${ }^{3}$ Fraunhofer Institute for Applied Information Technology (FhG-FIT), Schloss Birlinghoven, \\ Sankt Augustin, Germany \\ volker.wulfefit.fraunhofer.de
}

\begin{abstract}
We present the newly emerging research field of Computer Supported Collaborative Sports (CSCS). By applying innovative input and output technologies, it enables players to experience sportive activities in a shared computerized environment. Important dimensions in the design space of CSCS applications are discussed. Finally we present the FlyGuy, a prototypical realization of a CSCS device.
\end{abstract}

\section{Introduction}

Computer games have turned into a popular form of entertainment. An increasing number of people, specifically children, are playing computer games, thus converting them into an important leisure activity. When asked for the most fun entertainment activities in 2000, 35\% of Americans mentioned computer and video games outranging alternatives such as watching television, surfing in the internet, reading books, or going to cinema [6]. So computer games fascinate and suck people's attention.

However, the success of computer games has always been critically discussed. Unsuitable content of the games, social isolation of the players, and lacking physical activities are mayor critical concerns with regard to computer games.

Quite a number of computer games deal with shooting or killing activities. An often expressed criticism with regard to this type of games is based on the assumption that killing activities within shooting games will lead to an increased aggressive behavior in daily life [20]. While empirical investigations with regard to this hypothesis show heterogeneous results [5], the design of ethically less problematic but fascinating game content is a big challenge.

Critics have pointed out that intense use of computer games may lead to social isolation of the players [19]. However, social arrangements such as playing single user games in a group or LAN parties where multi user games are played in physical 
proximity may compensate for this problem. Certain computer games address this issue by allowing playing together across local distances.

Another problematic issue with regard to computer games is lacking physical activity when playing - which offers a strange contrast to the "physical" content of many games. The typical input devices of computer games are system-specific push buttons, different types of keyboards, joy sticks, or mice. Output is typically provided to the players by audio and graphical means (e.g. loudspeakers and screens of different types). Only recently with the emergence of Ubiquitous Computing new input and output technologies have come up [3]. Other approaches take given sportive activities like skateboarding and karate as a base and augment them with information technology. By doing so existing sports activities get an additional 'game content' [10], [17], [16].

In the following we want to get one step beyond by further integrating computer games and computer augmented sports. We will postulate the approach of Computer Supported Cooperative Sports (CSCS). By applying innovative input and output technologies we want to enable users to gain new experiences in a shared computerized game environment.

The paper is structured as follows. First we will present the state of the art in computer games with computer augmented sportive interfaces. Then we will outline the concept of Computer Supported Collaborative Sports. A prototype of this design paradigm, the FlyGuy approach to enable flights in a shared 3D space, will be presented. We will conclude by discussing our findings.

\section{Ubiquitous Games and Computer Augmented Sports}

Ubiquitous computing offers interesting new options to interact with computers through real world objects and spaces. They offer new opportunities to create innovative games and sportive input devices. The STARS environment offers a platform to realize different board games on a computer augmented table. Real world objects, such as chess figures, can be moved on the board and their positions can be tracked. Based on this input, a game engine can compute appropriate output behaviour [12]. Based on rather similar input technologies, Harvard and Lovind [8] have developed toys based on a rather different conceptual idea. They try to encourage storytelling by moving away from the computer screen and take physical objects (typically simple plastic toys) as an interface that permits to explore the quirks of a story. Stories can be recorded and attached to different toys and their actual position.

A different approach is taken by Sanneblad and Holmquist [21]. They distribute a game area on different hand-held computers in a way that the whole area can only be seen by means of all the different displays. The players have to move towards each other to perform activities, e.g. stiring PacMan in the classic arcade game, on those parts of the game area which are not represented on their own hand-held. In this case physical activities of the players result from the need to see the whole game area. 
Different approaches record human movements for navigation in virtual environments. Humphrey $\mathrm{II}^{1}$, developed by the Futurelab in Linz, is a flight simulator where the user emerges into a 3D virtual space by means of a head mounted display. The behaviour of an avatar representing the users can be controlled by means of arm movements. In the Virtual Fitness Center (Virku) an exercise bicycle is positioned in front of a video screen. The physical movements conducted on the exercise bicycle are used as input to modify the representation of 3D virtual environments from map information. Reversely, the map information affects the pedaling efforts. In an early implementation the players move this way along a hilly landscape in Finish Lapland [17].

Other approaches address collaborative sportive activities explicitly. They can be understood as early instances of CSCS research. $\mathrm{AR}^{2}$ is an augmented reality airhockey table with a virtual puck. The two players wear head-mounted displays to see a virtual puck on the table in front of them [18]. PingPongPlus is a system which augments traditional table tennis by means of a tracking device for the ball and a video projector. Different applications have been designed which project images on the table according to the location where the ball hits the table. When a ball hits the table in the water ripple mode, an image of a water ripple flows out from the spot the ball landed [10]. Mueller et al. [16] have developed a system which allows players to interact remotely through a life-size video conference screen using a regular soccer ball as an input device. Both players kick the ball against their local wall on which an audio and video connection with the other player is displayed. By tracking the position where the ball hits the wall specific single user games can be added on each player's side via an overlay technique.

While there are quite some interesting developments in the ubiquitous and entertainment computing fields, the sports engineering community has not come up with computer augmented sport devices. Most research is still restrained to analyse and model traditional sport devices or aspects of the human body (for a good summary see [23], [24]). There are few if any papers concerning the design of sport devices and none so far have touched the area of combining computers and sports in novel designs of sport equipments. If at all, respective contributions can be found in training science, but with the specific purpose to use the computer technology to achieve particular training objectives.

\section{Computer Supported Cooperative Sports}

Computer Supported Cooperative Sports investigates the design of computer applications which require sportive input activities to gain collective game experience. It is an interdisciplinary research field where sports engineers, computer scientists, designers, sport scientists, and social scientists need to cooperate, guided by a systematic design approach [15]. In the following we want to clarify the concept and discuss important aspects of the design space for innovative applications.

${ }^{1}$ www.aec.at/humphrey/ 


\subsection{Integrating Sports with Games}

In the following the hermeneutic and practical core of sports and games and their implications shall be identified, and related to one another. Sports in a traditional understanding has been defined as ,organized play that is accompanied by physical exertion, guided by a formal structure, organized within the context of formal and explicit rules of behaviour and procedures, and observed by spectators" ([2], p.143). Still widely spread, this formalizing definition coerces sports into a specific scheme and strangely strangles the scope for innovation with respect to social and individual use value. However, there are also more context-sensitive approaches, defining sports as a "specific expression of human movement behaviour" ([7], p.8) that becomes "sports" only by "a situation-specific reception and an attribution of meaning" ([9], p.34). Eventually it is the purpose an individual assigns to a movement which he considers being sportive (which in many cases encompass "physical exertion"), that defines sports. Reasons to do sports include fun, health, meeting with interesting people, maintaining fitness, and compensating for sedentary occupation [13], [14].

Doing sports and playing games have many similarities, especially the voluntary character of the activities motivated by a perception of fun. In the domain of computer games, sport genres have already been picked up. Players can simulate sport competitions, such as a soccer championship, on their computer. However, the aim of CSCS is not to simulate sports activities, but doing them.

\subsection{Input and Output Devices for Sports Activities}

An important dimension in the design space is obviously the type of sports activity which shapes the input and output interface to the computer augmented environment. Defining sports via the meaning individuals assign to a movement, one can imagine a wide scope of different activities. If we either presuppose the objective of fitness or at least like to reduce long-term physical harm, one of the essential requirements is balance in external load distribution, e.g. not to demand an over-utilization of the biceps and offering no stimulation to the triceps. Practical technical and biomechanical considerations and the wish to monitor progress furthermore suggest a reduction of movement complexity to a simple combination of translational and rotational movements - in which, however, one might have to compromise the natural feeling while moving around in a virtual world.

When becoming part of a computer augmented collaborative environment, existing sports may change their game idea altogether. Still less reference to an existing sport may finally be needed or wanted; save some examples where the similarity is explicitly aimed at (e.g. soccer in front of a video wall). So we can distinguish sport activities which already exist in the physical world from those which are newly designed within a computer-augmented environment.

With regard to the design of the input interface the question arises how to register sport activities appropriately. If this cannot be done by monitoring movements and forces in the device directly, e.g. the actual engine torque, then sensors of different kinds become an essential part of the design. These sensors can either measure the 
movements of the human body (e.g. stiring and pedaling an exercise bike) or of different types of sport tools (e.g. the ball in table tennis or the racket in hockey).

With regard to the design of the output interface in a distributed game environment one has to think of how to represent the activities of other actors and the physical texture of virtual space. This can either happen merely visually or also physically by means of forced feedback. For instance, in the Virku environment the physical texture of the virtual landscape translates into different levels of required pedaling efforts.

\subsection{Collaboration}

The concept of collaboration in CSCS environments needs some discussion. Sports, like many game genres, seem to imply competition either among individuals or among teams. However, in dancing or acrobatics it is the feeling of being together in combination with (joint) movements that people aim at. So in principle CSCS can be centred on cooperation or competition. Hence, the meaning of collaboration in CSCS can span the whole scope from multi user competitive settings (e.g. computeraugmented table tennis or a bicycles race in a virtual 3D environment), via settings of mere co-presence (e.g. playing soccer individually in a shared audio and video space or riding bicycles together in a virtual space) towards settings where cooperation is needed to achieve the common goals (e.g. moving in a game area distributed via different hand-helds or producing output loads that are converted into a stimulating input for the partner at a remote location).

From a computer science perspective, collaborative settings can be classified along the time-space dichotonomy [11]. With regard to the space, players in CSCS applications can either interact at the same place (e.g. computer augmented table tennis) or at remote locations (e.g. soccer within a shared media space). With regard to time, most of the applications in the field of entertainment computing are synchronous in the sense that the players interact with each other at the same time. However, asynchronous applications such as community systems may help shaping social relationships among players. Seay et al. [22] and Friedl [4] describe how synchronous applications of computer mediated communication such as chat or email can be integrated into Massive Multiplayer Online Games (MMOG). Friedl [4] stresses the importance of asynchronous features. Web pages allow, for instance, displaying information about player's performances in past games.

Another important dimension with regard to collaboration is the question whether the players know each other beforehand or whether they learn to know each other within the game environment. In the latter case specific technical features may be needed to introduce or match human actors [1]. Friedl [4] points out that personal information and information about players' performance and can stimulate social interactions. 


\subsection{Objectives and Vision}

CSCS emerges in an interesting intersection of sports, game and innovative technologies. It may help to tackle problems which are of imminent importance to individuals and the society as a whole:

- "Animated fitness equipment" will greatly enhance the motivation to do something for one's health and fitness, and to stick with it, by combining exertion with diversion (and diversity). It is further possible to address the wishes more directly and to pull the motivational triggers of different target groups.

- "Animated fitness worlds" will combine play, sports, and fitness: An overall leisure attraction may create an important offer to get kids away from stationary computer gaming, and thus to fight obesity and social isolation.

- "Computer controlled sports equipment" will allow monitoring movements and achievements, adapting training and rehabilitation, and enable remote supervision.

- "Computer enhanced sports equipment" may provide novel access to emotions and feelings, especially by combining movements and visual displays, which is not possible by purely mechancial sports equipment.

- "Computer supported collaborative sports equipment" will link people to do collaborative phyiscal activites; again enhancing motivation and opening up new physical communication channels for mates or even distributed teams.

To arrive at these objectives, however, puts high demands on how to conduct respective research and development projects. A project team heterogeneously assembled with engineers, computer scientists and sports experts will have to put their competences together, guided by a systematic approach to innovation in sports, and backed up by a distributed project management. A first pilot project in this area has been conducted and will be reported upon in the next section.

\section{The FlyGuy Approach}

We have developed a concept called FlyGuy ${ }^{2}$ for an innovative CSCS device which combines effective fitness training with playful challenges, social interaction, and a versatile entertainment. The work was conducted in a multidisciplinary design team which consisted of researchers and students from Germany, Japan, Mexico, and the United States.

\footnotetext{
2 The work on the FlyGuy was supported by the Japanese Ministry of Education in the project Tele Athletics New Experience (TANE). The work was conducted at the Technical University of Munich (Germany), Stanford University (USA), University of Siegen (Germany), Tokyo Institute of Technology (Japan), Tsukuba University (Japan), Universidad de las Americas (Puebla, Mexico), and University of Magdeburg (Germany).
} 

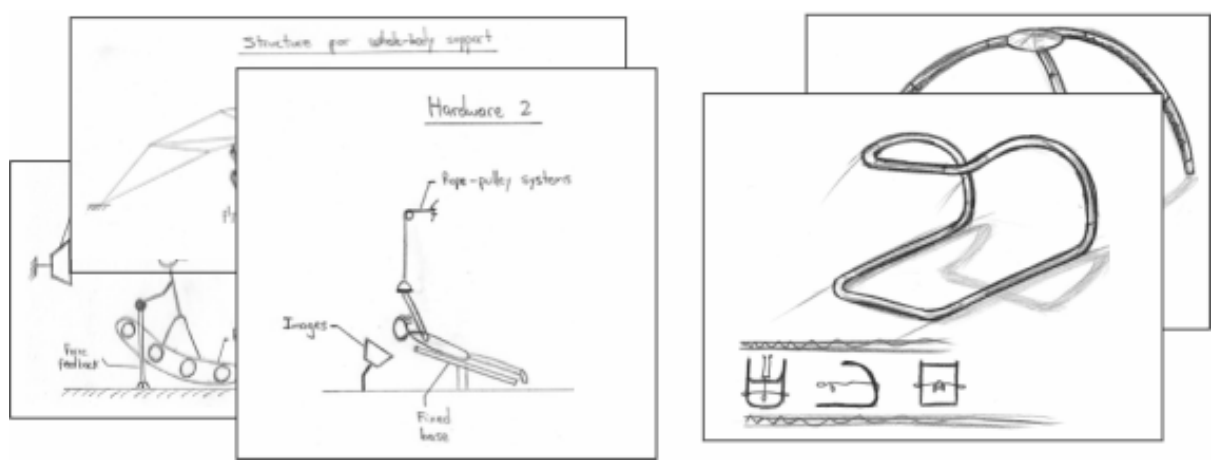

Fig. 1. First sketches of the FlyGuy and design alternatives of the frame which holds the human actor when flying

Other than PingPongPlus or the soccer game at the video wall, we wanted to design a collaborative environment for new physical experiences and sports activities. In a first face-to-face meeting of the project team, flying was identified as an interesting sports activity which humans can only experience in a computer augmented environment or by means of specific avionic devices such as hang gliders.

Our concept looks as follows: The player immerses via a head mounted display into a 3D virtual environment. It controls a flight simulation by its body motion. In a first explorative realization two handles are grabbed with the hands; the flight direction can be changed via rotation of the torso, the height by pulling or pushing a lever horizontally. One of the reasons we chose to realize the flight movement in this "starfighter" fashion was that it appeared the most natural way to the test persons we asked to "fly" on a small table structure. Bird-like movements were less frequent and above all would be more difficult to realize and to put into an efficient training regimen. The training effect is intensified by providing resistance for both concentric and excentric movements; thus it is possible to realize extreme intensity and early exhaustion. In further stages we plan to include the leg movements for acceleration and deceleration. The motions are captured by sensors located in the joints of the lever structure and transformed into electrical signals which are then being transmitted to a microcontroller and PC. These data are used as input to control the flight simulation which is perceived by the player via a head mounted display. The player so far is hanging in a frame made of aluminum similar to the frame of a hang glider.

In the virtual space, the sportsman has the possibility to solve different flight tasks and meet other persons and fly and exercise with them, even if they are in a far distant location in the real world. Whenever the players reach certain proximity in the virtual space, an audio channel is opened. Therefore different FlyGuy devices are connected via a local network or internet. The underlying technical infrastructure for communication is based on a dedicated server model architectures because it combines some peer-2-peer ideas with a client-server model and it will be possible to integrate an single-user or training modus.

For creating the virtual environment, we explored different popular 3D game engines and finally opted to tailor an existing game like Half-Life II for our purpose. 
This also helped to overcome the problem to arrive at a critical mass for efficient useage, as it makes it easier to integrate other players, which do not have the FlyGuy device, but nevertheless can use the FlyGuy game as well.

What mostly differentiates the FlyGuy from Humphrey II (Chapter 2) is the aspect that FlyGuy is a device for efficient fitness training, while Humphrey II focuses on entertainment only. This becomes apparent when looking at the rather non ergonomic pattern for user input. Moreover, Humphrey II is a single user environment which does not realize any collaborative feature.
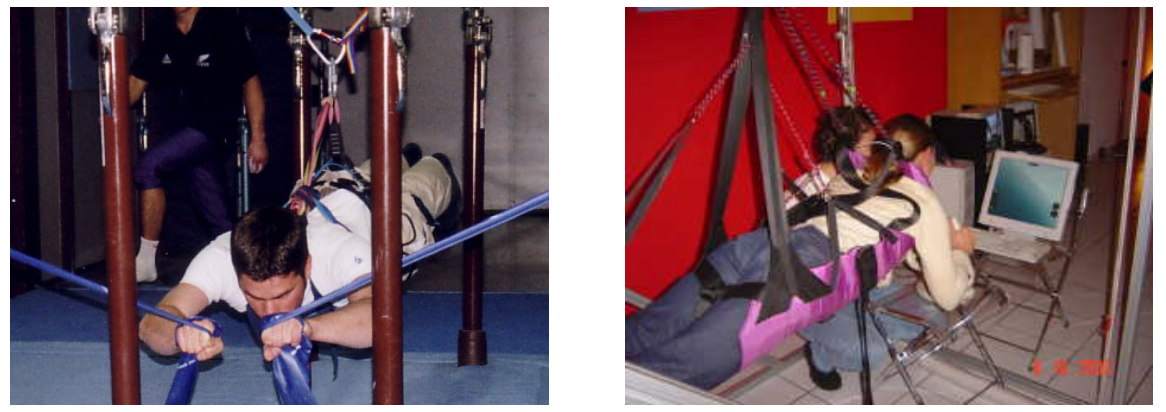

Fig. 2. Experimenting with early prototypes of the FlyGuy

After defining the concept, the team separated again and worked on its realization (mechanics, mechatronics, network structure, virtual environment, output devices, biomechanics, game plan, sports scientific aspects, etc.) in a distributed fashion.

In a second face-to-face meeting a functional prototype was assembled and tested. This prototype was built to explore technical design issues such as the location of the hanging and the fix of the lever structure. We also tested whether the data conversion worked the way it was anticipated. Further functional design issues were explored such as the steering mechanisms. We also investigated which degree of freedom and what kind of support are necessary to provide the basis for efficient and safe training.

We evaluated our concepts internally during the design process. It turned out that some aspects of the prototype's design worked out better than others. For example the horizontal flight posture and the steering of the flight simulation were rated well and very intuitive, while the usage of a fixed and stiff lever structure was regarded suboptimal because it does not perfectly match the idea of "free" flight motions. Other aspects which need to be improved are the overly complicated access into the device and lacking adaptability with regard to different user anthropometries. We could not yet evaluate the game software and the virtual environment, as these were still rudimentarily adapted versions of software produced for completely different purposes.

\section{Conclusion}

We have presented the concept of Computer Supported Collaborative Sports and presented the FlyGuy, a first prototypical realization of a CSCS device. The FlyGuy 
prototype stimulated considerable interest from test users as well as from partners from industry. Obviously the attempt to transfer the excitement of computer games into a motivation for fitness training and thereby even allowing joint physical activities by partners far apart will mark an important trend in the future of entertainment computing and fitness-oriented sports alike.

The introduction of computers into cooperative sports equipment and settings does not only offer new areas of application for computers in entertainment but also opens up new dimensions in sports and fitness:

- There is a whole array of novel means to increase motivation to participate in sportive or health-sustaining activities.

- Linked via internet, people in different locations can do sports, share physical fun or follow rehabilitation schemes together.

- The development of virtual worlds and connected input-output devices provide for sensoric and emotional sensations that cannot be experienced otherwise; "flying" being just one example.

To explore this line of thoughts furthermore, we will need to realize a variety of different CSCS devices. Empirical evaluations of their appropriation by various groups of players will help us to even better understand the design space lined out in this paper.

\section{References}

1. Al-Zubaidi, K., Stevens, G.: CSCP at Work, in Proceedings of the Conference Mensch und Computer 2004 (MC 2004), Teubner, Stuttgart 2004 in press

2. Anshel, M.A. (Ed.): Dictionary of the Sports and Exercise Sciences; Human Kinetics Books, Illinois 1991

3. Björk, S.; Holopainen, J.; Ljundstrand, P.; Mandryk, R. (eds): Special Issue on Ubiquitous Games, in: Personal and Ubiquitous Computing, Vol. 6, 2002, pp. 358 - 361

4. Friedl, M.: Online Game Interactivity Theory. Hingham, Charles River Media 2003

5. Fritz, J.; Fehr, W. (eds): Handbuch Medien und Computer-Spiele, Bonn 1997

6. IDSA: Interactive Digital Software Association, http://www.idsa.com

7. Haag, H. (ed.): Sportphilosophie; Verlag Karl Hofmann, Schorndorf 1996

8. Harvard, Å., Løvind, S.: "Psst"-ipatory Design. Involving artists, technologists, students and children in the design of narrative toys, in: Binder, T., Gregory, J., Wagner, I. (eds) Proceedings of the PDC 2002 Participatory Design Conference, Malmö, 2002

9. Heinemann, K.: Einführung in die Soziologie des Sports; Verlag Karl Hofmann, Schorndorf 1998

10. Ishii; H.; Wisneski, C.; Orbanes, J.; Chun, B.; Paradiso, J.: PingPongPlus: Design of an Athletic-Tangible Interface for Computer-Supported Cooperative Play, in: Proceddings of CHI'99, May 15 - 20, 1999, ACM-Press, New York, pp. 394 - 401

11. Johansen, R.: Current User Approaches to Groupware, in Johansen, Robert (ed): Groupware, Freepress, New York 1988, pp. 12-44

12. Magerkurth, C.; Stenzel, R.: Computerunterstütztes kooperatives Spielen - Die Zukunft des Spieltisches, in: Ziegler, J.; Szwillus, G. (eds): Proceedings of Mensch \& Computer 2003 (MC '03), Stuttgart, September 7-10, 2003, Teubner 
13. Meyer, M.: Zur Entwicklung der Sportbedürfnisse; Dissertation, German Sports University, Cologne 1992

14. Moritz, E.F., Steffen, J.: Test For Fun - ein Konzept für einen nutzerorientierten Sportgerätetest; in: Roemer et al (eds) Sporttechnologie zwischen Theorie und Praxis; Shaker Verlag, Aachen 2003, pp. 43-63

15. Moritz, E.F.: Systematic Innovation in Popular Sports, in: $5^{\text {th }}$ Conference of the International Sports Engineering Association, September 14-17, 2004, Davis CA, in press

16. Mueller, F.; Agamanolis, S.; Picard, R.: Exertion Interface: Sports over a Distance for Social Bonding and Fun, in: Proceedings of CHI 2003, April 5 - 10, 1999, ACM-Press, New York, pp. $561-568$

17. Mokka, S.; Väätänen, A.; Välkkynen, P.: Fitness Computer Games with a Bodily User Interface, in: Proceedings of the Second International Conference on Entertainment Computing, Pittsburgh, Pennsylvania, May 8 - 10, 2003, ACM International Conference Proceeding Series, pp 1-3

18. Ohshima, T.; Satoh, K.; Yamamoto, H.; Tamura, H.: AR2 Hockey, in: Conference Abstracts and Applications of SIGGRAPH'98, ACM-Press, New York, p. 110

19. Provenzo, Eugene F.: Video Kids: Making Sense of Nintendo. Harvard University Press Cambridge, MA 1991

20. Rauterberg, M.: Emotional Aspects of Shooting Activities: 'Real' versus 'Virtual' Actions and Targets, in: Proceedings of 2nd International Conference on Entertainment Computing (ICEC 2003); May 8-10, 2003, Pittsburgh, PA, ACM Digital Library

21. Sanneblad, J., Holmquist, L E.: Designing Collaborative Games on hand-held Computers, in: Proceedings of the SIGGRAPH 2003 conference on Sketches \& applications: in conjunction with the 30th annual conference on Computer graphics and interactive techniques, San Diego, CA, July 27 - 31, 2003, ACM-Press, New York 2003

22. Seay, A. F., W. J. Jerome, et al.: Project Massive 1.0: Organizational Commitment, Sociability and Extraversion in Massively Multiplayer Online Games. LEVEL UP Digital Games Research Conference, Utrecht University 2003

23. Subic, A.J., Haake, S.J. (eds): The Engineering of Sport 3; Blackwell Science, Cambridge 2000

24. Ujihashi, S., Haake, S.J. (eds): The Engineering of Sport 4; Blackwell Science, Cambridge 2002 